\title{
On some properties of Generalized Transmuted - Kumaraswamy Distribution
}

\author{
Aliyu Ismail Ishaq \\ Department of Statistics, Faculty of Physical Sciences, \\ Ahmadu Bello University, Zaria - Nigeria. \\ Email: binishaq05@gmail.com \\ Abubakar Usman \\ Department of Statistics, Faculty of Physical Sciences, \\ Ahmadu Bello University, Zaria - Nigeria. \\ Email: abubakarusman28@gmail.com \\ Tasiu Musa \\ Department of Statistics, Faculty of Physical Sciences, \\ Ahmadu Bello University, Zaria - Nigeria. \\ Email: Mtasiu@abu.edu.ng \\ Samson Agboola \\ Department of Statistics, Faculty of Physical Sciences, \\ Ahmadu Bello University, Zaria - Nigeria. \\ Email: abuagboola@gmail.com
}

\begin{abstract}
This article introduces a new lifetime model called the generalized transmuted-Kumaraswamy distribution which extends the Kumaraswamy distribution from the family proposed by Nofal et al., (2017). We provided hazard and survival functions of the proposed distribution. The statistical properties of the proposed distribution are provided and the method of Maximum Likelihood Estimation (MLE) was proposed in estimating its parameters.
\end{abstract}

Keywords: Generalized Transmuted - Kumaraswamy distribution, hazard function, moment generating function, survival function and order statistics.

\section{Introduction}

The Kumaraswamy probability distribution was pioneered by Ponnambalam Kumaraswamy (often known as Poondi Kumaraswamy, 1980) is a family of continuous probability distributions defined on the interval $[0,1]$ suitable for physical variables that have lower and upper bounded of both its probability density function and cumulative distribution function. The Kumaraswamy (1980) distribution is a similar to Beta distribution, but much simpler to use especially in simulation studies. This distribution has a wide range of applications in hydrology which faithfully fit hydrological random variables such as daily rainfall, daily stream flow, etc. and many natural phenomena. For more details see Sundar and Subbiah (1989), Fletcher and Ponnambalam (1996), Seifi (2000), Ponnambalam (2001) and Ganji (2006). The kumaraswamy probability density function has the same basic shape properties as the beta distribution (Jones, 2009) and Cordeiro $(2010,2012)$ but it depends on the values of its parameters: it is unimodal for c $>1$ and $\mathrm{d}>1$; uniantimodal for $\mathrm{c}<1$ and $\mathrm{d}<1$; increasing for $\mathrm{c}>1$ and $\mathrm{d} \leq 1$; decreasing for $\mathrm{c} \leq 1$ and $\mathrm{d}>1$ and constant for $\mathrm{c}=\mathrm{d}=1$. Jones (2009), made some similarities and 
differences between the kumaraswamy and beta distributions and also highlighted several advantages of kumaraswamy distribution over beta distribution such as a simple normalizing constant; simple explicit formulae for the distribution and quantile functions which do not involve any special functions; a simple formula for random variate generation; explicit formulae for moments of order statistics. The cumulative distribution function (cdf) and probability density function (pdf) of the Kumaraswamy distribution are respectively given by

$$
G(x ; c, d)=\int_{0}^{x} g(t ; c, d) d t=c d \int_{0}^{x} t^{c-1}\left(1-t^{c}\right)^{d-1} d t=1-\left(1-x^{c}\right)^{d}, x \in(0,1)
$$

and

$$
g(x ; c, d)=c d x^{c-1}\left(1-x^{c}\right)^{d-1}, \quad x \in(0,1)
$$

where $c, d$ are positive shape parameters.

Shaw and Buckley (2007) proposed a new transmuted family using Quadratic rank transmutation map which was proposed and studied by Arya (2011), Elbatal (2013), Ahmad (2015) and many others have also been defined and studied in the literatures. In this research we introduce a new lifetime model which is generalized two-parameter Kumaraswamy distribution, called the generalized transmuted - Kumaraswamy (GT-Kw) distribution which extends the quadratic rank transmutation map pioneered by Shaw (2007) by adding two additional shape parameters to generate more flexible distributions based on Generalized Transmuted - G ( GT - G) family pioneered by Nofal et al.,(2017).

Let $G(x, \zeta)$ and $g(x, \zeta)$ denote the cumulative distribution and density functions of baseline model with parameter vector $\zeta$. The cdf and pdf of GT - G family defined by Nofal (2017) are given by:

$$
F(x ; \lambda, a, b, \zeta)=F(x)=G(x, \zeta)^{a}\left[(1+\lambda)-\lambda G(x, \zeta)^{b}\right], \quad x \in \mathbb{R}
$$

and

$$
f(x ; \lambda, a, b, \zeta)=f(x)=g(x, \zeta) G(x, \zeta)^{a-1}\left[a(1+\lambda)-\lambda(a+b) G(x, \zeta)^{b}\right], \quad x \in \mathbb{R}
$$

where $a, b$ are positive shape parameters and $|\lambda| \leq 1$ is transmuting parameter. Now, equation (4) can also be written as:

$$
\begin{aligned}
f(x ; \lambda, a, b, \zeta) & =a(1+\lambda) g(x, \zeta) G(x, \zeta)^{a-1}-\lambda(a+b) g(x, \zeta) G(x, \zeta)^{a+b-1} \\
& =(1+\lambda) h_{a}(x)-\lambda h_{a+b}(x)
\end{aligned}
$$

which is a mixture of two E-G densities with two power parameters a and $(a+b)$, where $h_{a}(x)=a g(x, \zeta) G(x, \zeta)^{a-1}$ and $h_{a+b}(x)=(a+b) g(x, \zeta) G(x, \zeta)^{a+b-1}$.

A random variable $X$ with pdf (4) is denoted by $\operatorname{GTKw}(x ; \lambda, a, b, \zeta)$. Nofal (2017) proposed and studied generalized transmuted - Weibull distribution in the same way, we are generalizing Kumaraswamy distribution. Other research can be mentioned such as generalized transmuted - Rayleigh by Ishaq (2018), generalized transmuted - generalized Rayleigh distributions by Usman (2018) and many more. The GT - G family reduces to some family of distributions when the parameters change. If $b=\lambda=0$, it reduces to $\mathrm{E}$ - 
G family (Gupta, 1998) and if $a=b=1$, the GT - G family corresponds to $\mathrm{T}-\mathrm{G}$ family (Shaw, 2007).

\section{Expansion for the distribution and density functions}

A random variable $X$ is said to have Generalized Transmuted - Kumaraswamy (GT-Kw) distribution denoted by $\operatorname{GTKw}(x ; \lambda, a, b, c, d)$ with parameters $a, b, c, d>0$ and $|\lambda| \leq 1$ if its cdf and pdf are given by

$$
F(x)=\left(1-\left(1-x^{c}\right)^{d}\right)^{a}\left[(1+\lambda)-\lambda\left(1-\left(1-x^{c}\right)^{d}\right)^{b}\right], \quad x \in(0,1)
$$

and

$$
f(x)=c d x^{c-1}\left(1-x^{c}\right)^{d-1}\left(1-\left(1-x^{c}\right)^{d}\right)^{a-1}\left[a(1+\lambda)-\lambda(a+b)\left(1-\left(1-x^{c}\right)^{d}\right)^{b}\right], \quad x \in(0,1)
$$

respectively.

Using (7), the proposed density function can also be written as a mixture of two E-G densities defined as

$f(x)=a(1+\lambda) c d x^{c-1}\left(1-x^{c}\right)^{d-1}\left(1-\left(1-x^{c}\right)^{d}\right)^{a-1}-\lambda(a+b) c d x^{c-1}\left(1-x^{c}\right)^{d-1}\left(1-\left(1-x^{c}\right)^{d}\right)^{a+b-1}$

Consider the binomial expansion to the last term of (8)

$$
\left.\left(1-\left(1-x^{c}\right)^{d}\right)^{a+b-1}=\sum_{i=0}^{\infty}(-1)^{i}{ }_{i}^{a+b-1}\right)\left(\left(1-x^{c}\right)^{d}\right)^{i}
$$

Substituting (9) in (8) we can have

$$
\left.\left.=a(1+\lambda) c d x^{c-1} \sum_{i=0}^{\infty}(-1)^{i}{ }_{i}^{a-1}\right)\left(1-x^{c}\right)^{d(i+1)-1}-\lambda(a+b) c d x^{c-1} \sum_{i=0}^{\infty}(-1){ }^{i}{ }_{i}^{a+b-1}\right)\left(1-x^{c}\right)^{d(i+1)-1}
$$

Also, applying (9) in (10) we get

$$
\begin{aligned}
& =a(1+\lambda) c d \sum_{i, j=0}^{\infty}(-1)^{i+j}\left(\begin{array}{c}
a-1 \\
i
\end{array}\right)\left(\begin{array}{c}
d(i+1)-11 \\
j
\end{array}\right) x^{c(i+j)-1}-\lambda(a+b) c d \sum_{i, j=0}^{\infty}(-1)^{i+j}\left(\begin{array}{c}
a+b-1 \\
i
\end{array}\right)\left(\begin{array}{c}
d(i+1)-11 \\
j
\end{array}\right) x^{c(i+j)-1} \\
f(x) & =c d \sum_{i, j=0}^{\infty}(-1)^{i+j}\left(\begin{array}{c}
a-1 \\
i
\end{array}\right)\left(\begin{array}{c}
d(i+1)-11 \\
j
\end{array}\right) x^{c(i+j)-1}\left[a(1+\lambda)\left(\begin{array}{c}
a-1 \\
i
\end{array}\right)-\lambda(a+b)\left(\begin{array}{c}
a+b-1 \\
i
\end{array}\right)\right]
\end{aligned}
$$

Plots of the cdf and pdf of the proposed distribution for some parameters are displayed in figure 1 . 
cdf of GTKWD

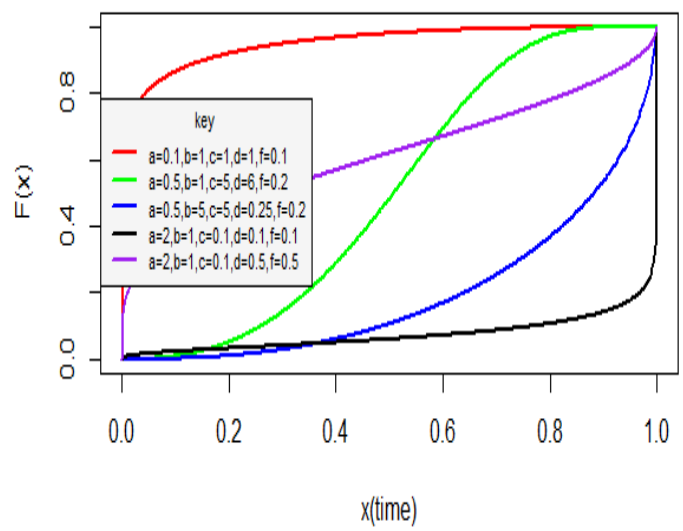

(a) pdf of GTKWD

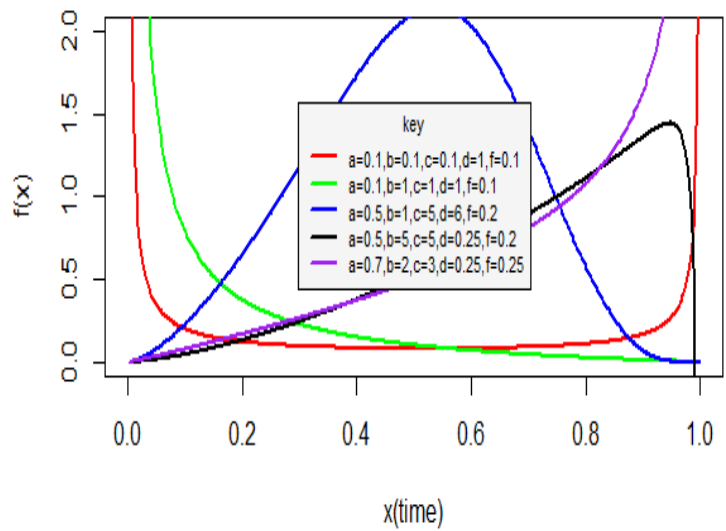

(b)

Figure 1: (a) and (b) are plots of cdf and pdf of GT-Kw distribution at $\lambda=f$ for some parameter values.

\section{Survival and Hazard functions \\ 3.1 Survival function}

The survival function of the GT-Kw distribution with parameters $a, b, c, d>0$ and $|\lambda| \leq 1$ is given as

$$
S(x)=1-F(x)=1-\left(1-\left(1-x^{c}\right)^{d}\right)^{a}\left[(1+\lambda)-\lambda\left(1-\left(1-x^{c}\right)^{d}\right)^{b}\right], x \in(0,1)
$$

where $F(x)$ is defined in (6)

\subsection{Hazard function}

The hazard function of the proposed distribution is given by

$$
H(x)=\frac{f(x)}{S(x)}=\frac{c d x^{c-1}\left(1-x^{c}\right)^{d-1}\left(1-\left(1-x^{c}\right)^{d}\right)^{a-1}\left[a(1+\lambda)-\lambda(a+b)\left(1-\left(1-x^{c}\right)^{d}\right)^{b}\right]}{1-\left(1-\left(1-x^{c}\right)^{d}\right)^{a}\left[(1+\lambda)-\lambda\left(1-\left(1-x^{c}\right)^{d}\right)^{b}\right]}, x \in(0,1)
$$

where $a, b, c, d>0$ are shape parameters, $|\lambda| \leq 1$ is the transmuting parameter and $\mathrm{f}(\mathrm{x})$, $\mathrm{S}(\mathrm{x})$ are density and survival functions defined in (7) and (12) respectively. 
Plots of the survival and hazard functions of the proposed GT-Kw distribution are presented in Figure 2.

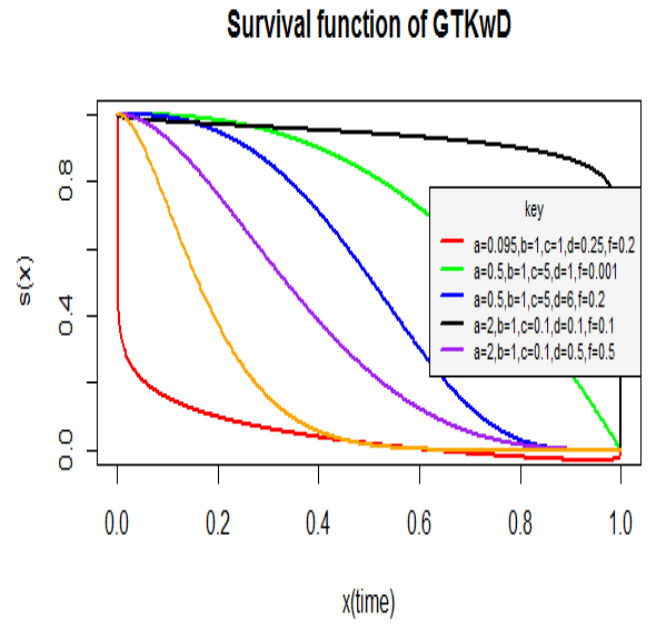

(c)

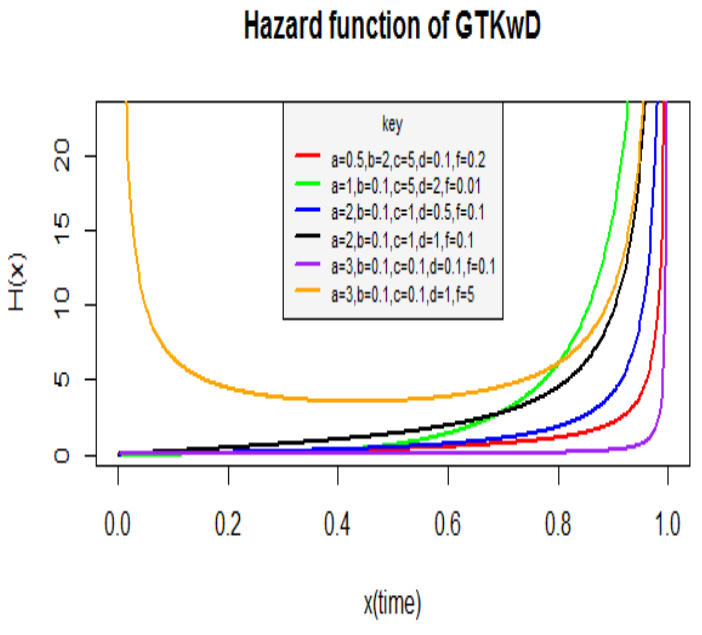

(d)

Figure 2: (c) and (d) are plots of survival and hazard functions of the GT-Kw distribution at $\lambda=f$ for some parameter values.

\section{Statistical Properties of the GT-Kw distribution}

In this section, some properties of the proposed distribution were studied such as moments, moment generating function, characteristic function, incomplete moment and order statistics.

\subsection{Moments}

Theorem 1: If $X$ has the $\operatorname{GTKw}(x ; \lambda, a, b, c, d)$ distribution with $|\lambda| \leq 1$, then the $\mathrm{r}^{\text {th }}$ moments of $X$ is given by

$$
E\left(X^{r}\right)=d \sum_{i=1}^{\infty}(-1) \beta\left(\frac{r}{c}+1, d(1+i)\right)\left[a(1+\lambda)\left(\begin{array}{c}
a-1 \\
i
\end{array}\right)-(a+b) \lambda\left(\begin{array}{c}
a+b-1 \\
i
\end{array}\right)\right]
$$

Proof: Let $X$ has a $\operatorname{GTKw}(x ; \lambda, a, b, c, d)$ distribution, then the $\mathrm{r}^{\text {th }}$ moments of $X$ proposed by Nofal (2017) is defined as

$$
E\left(X^{r}\right)=(1+\lambda) E\left(Y_{a}^{r}\right)-\lambda E\left(Y_{(a+b)}^{r}\right)
$$

But,

$$
E\left(Y_{a}^{r}\right)=\int_{-\infty}^{\infty} x^{r} h_{a}(x) d x=a c d \int_{0}^{1} x^{c+r-1}\left(1-x^{c}\right)^{d-1}\left(1-\left(1-x^{c}\right)^{d}\right)^{a-1} d x
$$

Using generalized binomial theorem, equation (15) can be written as 


$$
=\operatorname{acd} \sum_{i=0}^{\infty}(-1)^{i}{ }_{i}^{a-1} \int_{0}^{1} x^{c+r-1}\left(1-x^{c}\right)^{d(1+i)-1} d x
$$

Let $y=x^{c}$ and $x=y^{1 / c} \Rightarrow d x=\frac{d y}{c x^{c-1}}$

Inserting (17) in (16) we have

$$
\begin{aligned}
& =\operatorname{acd} \sum_{i=0}^{\infty}(-1)^{i}\left(\begin{array}{c}
a-1 \\
i
\end{array}\right) \int_{0}^{1} y^{r / c}(1-y)^{d(1+i)-1} d y \\
& =a d \sum_{i=0}^{\infty}(-1)^{i}\left(\begin{array}{c}
a-1 \\
i
\end{array}\right) \beta\left(\frac{r}{c}+1, d(1+i)\right)
\end{aligned}
$$

and

$$
\begin{aligned}
E\left(Y_{(a+b)}^{r}\right) & =\int_{-\infty}^{\infty} x^{r} h_{(a+b)}(x) d x=(a+b) c d \int_{0}^{1} x^{c+r-1}\left(1-x^{c}\right)^{d-1}\left(1-\left(1-x^{c}\right)^{d}\right)^{a+b-1} d x \\
& =d(a+b) \sum_{i=0}^{\infty}(-1){ }^{i}\left(\begin{array}{c}
a+b-1 \\
i
\end{array}\right) \beta\left(\frac{r}{c}+1, d(1+i)\right)
\end{aligned}
$$

Finally, the $\mathrm{r}^{\text {th }}$ moments of the proposed GT-Kw distribution is obtained by substituting (18) and (19) in (14) as given in (20)

$$
\begin{gathered}
\left.E\left(X^{r}\right)=(1+\lambda)\left[a d \sum_{i=0}^{\infty}(-1){ }_{i}^{i}{ }_{i}^{a-1}\right) \beta\left(\frac{r}{c}+1, d(1+i)\right)\right]-\lambda\left[\left.d(a+b) \sum_{i=0}^{\infty}(-1)\right|_{i} ^{i+b-1}{ }_{i}^{a} \beta\left(\frac{r}{c}+1, d(1+i)\right)\right] \\
=d \sum_{i=0}^{\infty}(-1) \beta\left(\frac{r}{c}+1, d(1+i)\right)\left[a(1+\lambda)\left(\begin{array}{c}
a-1 \\
i
\end{array}\right)-(a+b) \lambda\left(\begin{array}{c}
a+b-1 \\
i
\end{array}\right)\right]
\end{gathered}
$$

which completes the proof.

\subsection{Moment Generating Function (MGF)}

Theorem 2: If $X$ has the $G T K w(x ; \lambda, a, b, c, d)$ distribution with $|\lambda| \leq 1$, then the MGF of $X$ is given by

$$
M(t)=\sum_{r=0}^{\infty} \frac{t^{r}}{r !}\left\{d \sum_{i=0}^{\infty}(-1) \beta\left(\frac{r}{c}+1, d(1+i)\right]\left[a(1+\lambda)\left(\begin{array}{c}
a-1 \\
i
\end{array}\right)-(a+b) \lambda\left(\begin{array}{c}
a+b-1 \\
i
\end{array}\right)\right]\right\}
$$

Proof: Let $\mathrm{X}$ has a $\operatorname{GTKw}(x ; \lambda, a, b, c, d)$ distribution, then the MGF of $X$ proposed by Nofal (2017) is defined as

$$
M(t)=(1+\lambda) M_{a}(t, \zeta)-\lambda M_{(a+b)}(t, \zeta)
$$

Where $M_{\rho}(t, \zeta)$ is the generating function of $Y_{\rho}$ for $\rho=a, a+b$.

Therefore,

$\left.M_{a}(t, c, d)=\int_{-\infty}^{\infty} e^{t x} h_{a}(x) d x=\sum_{r=0}^{\infty} \frac{t^{r}}{r !}\left[a d \sum_{i=0}^{\infty}(-1)^{i}{ }_{i}^{a-1}\right) \beta\left(\frac{r}{c}+1, d(1+i)\right)\right]$ 
where $e^{t x}=\sum_{r=0}^{\infty} \frac{(t x)^{r}}{r !}$

Also,

$$
\left.M_{(a+b)}(t, c, d)=\sum_{r=0}^{\infty} \frac{t^{r}}{r !}\left[d(a+b) \sum_{i=0}^{\infty}(-1){ }^{i}{ }_{i}^{a+b-1}\right) \beta\left(\frac{r}{c}+1, d(1+i)\right)\right]
$$

By substituting (22) and (23) in (21) we have the proposed MGF of GT-Kw distribution given by

$M(t)=\sum_{r=0}^{\infty} \frac{t^{r}}{r !}\left\{d \sum_{i=0}^{\infty}(-1) \beta\left(\frac{r}{c}+1, d(1+i)\right)\left[a(1+\lambda)\left(\begin{array}{c}a-1 \\ i\end{array}\right)-(a+b) \lambda\left(\begin{array}{c}a+b-1 \\ i\end{array}\right)\right]\right\}$

which complete the proof.

\subsection{Characteristics Function}

The characteristics function of the proposed GT-Kw distribution is given by 5

$\varphi_{X(t)}=\int_{-\infty}^{\infty} e^{i t x} f(x) d x=\sum_{r=0}^{\infty}\left(\frac{i t}{r !}\right)^{r} \int_{0}^{\infty} x^{r}\left((1+\lambda) h_{a}(x)-\lambda h_{a+b}(x)\right) d x=\sum_{r=0}^{\infty}\left(\frac{i t}{r !}\right)^{r} E\left(X^{r}\right)(25)$

\subsection{Incomplete moments}

Theorem 3: The $\mathrm{r}^{\text {th }}$ incomplete moments, say $M_{r}(y)$ of the GT-Kw distribution is given by

$$
M_{r}(y)=d \sum_{i=0}^{\infty}(-1) \beta_{y}\left(\frac{r}{c}+1, d(1+i)\right)\left[a(1+\lambda)\left(\begin{array}{c}
a-1 \\
i
\end{array}\right)-(a+b) \lambda\left(\begin{array}{c}
a+b-1 \\
i
\end{array}\right)\right]
$$

Proof: If $X$ has $G T-K w$ distribution, then the $\mathrm{r}^{\text {th }}$ incomplete moments of $X$ proposed by Nofal (2017) is defined as

$$
M_{r}(y)=\int_{-\infty}^{y} x^{r} f(x) d x=(1+\lambda) M_{r, a}(y)-\lambda M_{r, a+b}(y)
$$

Let us consider,

$$
\begin{aligned}
M_{r, a}(y) & =\int_{-\infty}^{y} x^{r} h_{a}(x) d x=a c d \int_{0}^{y} x^{c+r-1}\left(1-x^{c}\right)^{d-1}\left(1-\left(1-x^{c}\right)^{d}\right)^{a-1} d x \\
& =a c d \sum_{i=0}^{\infty}(-1)^{i}\left(\begin{array}{c}
a-1 \\
i
\end{array} \int_{0}^{y} x^{c+r-1}\left(1-x^{c}\right)^{d(i+1)-1} d x\right.
\end{aligned}
$$

Applying (17) in (27) becomes

$$
\begin{aligned}
& =a d \sum_{i=0}^{\infty}(-1)^{i}\left(\begin{array}{c}
a-1 \\
i
\end{array}\right) \int_{0}^{y} y^{r / c}(1-y)^{d(1+i)-1} d y \\
& =a d \sum_{i=1}^{\infty}(-1)^{i}\left(\begin{array}{c}
a-1 \\
i
\end{array}\right) \beta_{y}\left(\frac{r}{c}+1, d(1+i)\right)
\end{aligned}
$$

and, 


$$
M_{r,(a+b)}(y)=\int_{-\infty}^{y} x^{r} h_{(a+b)}(x) d x=d(a+b) \sum_{i=0}^{\infty}(-1)^{i}\left(\begin{array}{c}
a+b-1 \\
i
\end{array}\right) \beta_{y}\left(\frac{r}{c}+1, d(1+i)\right)
$$

Finally, put (28) and (29) in (26) we can have a proposed $\mathrm{r}^{\text {th }}$ incomplete moments as given in (30)

$M_{r}(y)=d \sum_{i=0}^{\infty}(-1) \beta_{y}\left(\frac{r}{c}+1, d(1+i)\right)\left[a(1+\lambda)\left(\begin{array}{c}a-1 \\ i\end{array}\right)-(a+b) \lambda\left(\begin{array}{c}a+b-1 \\ i\end{array}\right)\right]$

\subsection{Order Statistics}

Let $X_{1}, X_{2}, \ldots \ldots . ., X_{n}$ be a random sample drawn from GT-Kw random variables.

Let $X_{(1)}, X_{(2)}, \ldots \ldots . . ., X_{(n)}$ denoted the order statistics from these $\mathrm{n}$ variables. The pdf $f_{i, n}(x)$ of the $i^{\text {th }}$ order statistics for a random sample $X_{(i)}$ is given by:

$$
f_{i, n}(x)=\frac{n !}{(i-1) !(n-i) !} f(x) F(x)^{i-1}[1-F(x)]^{n-i}
$$

where $\mathrm{F}(\mathrm{X})$ and $\mathrm{f}(\mathrm{x})$ are defined in (6) and (7) respectively. Applying binomial expansion to the last term of (31) we have

$$
\begin{aligned}
f_{i, n}(x) & \left.=\frac{n !}{(i-1) !(n-i) !} f(x) \sum_{k=0}^{n-i}(-1)^{k}{ }_{k}^{n-i}\right) F(x)^{i+k-1} \\
& =\frac{n !}{(i-1) !(n-i) !} f(x) \sum_{k=0}^{n-i}(-1)^{k}{ }_{k}^{n-i}{ }_{k} \sum_{l=0}^{\infty} w_{l} G(x)^{b l+a(i+k-1)}
\end{aligned}
$$

but $w_{l}=(-1)^{l}\left(\begin{array}{c}i+k-1 \\ l\end{array}\right) s^{l}(1+\lambda)^{i+k-1}$ and $s=\lambda / 1+\lambda$. Equation (32) can also be written as

$$
f_{i, n}(x)=\sum_{k=0}^{n-i} \sum_{l=0}^{\infty} w_{l}^{*}\left[\psi h_{b l+a(i+k)}(x)-\omega h_{b(1+l)+a(i+k)}(x)\right]
$$

which is $i^{\text {th }}$ order statistics proposed by Nofal (2017), where $w_{l}^{*}=\frac{n !(-1)^{k}}{(i-1) !(n-i) !} w_{l}\left(\begin{array}{c}n-i \\ k\end{array}\right)$, $\psi=\frac{a(1+\lambda)}{b l+a(i+k)}$ and $\omega=\frac{\lambda(a+b)}{b(1+l)+a(i+k)}$.

The density function in (33) revealed that, the order statistics has a mixture of two E-G densities with two power parameters $b l+a(i+k)$ and $b(1+l)+a(i+k)$ respectively. The $i^{\text {th }}$ order statistics of the GT-Kw distribution is given by

$f_{i, n}(x)=\sum_{k=0}^{n-i} \sum_{l, m=0}^{\infty} c d x^{c-1}\left(1-x^{c}\right)^{d(1+m)-1}(-1)^{m} w_{l}^{*}\left[a(1+\lambda)\left(\begin{array}{c}b l+a(i+k)-1 \\ m\end{array}\right)-\lambda(a+b)\left(\begin{array}{c}b(1+l)+a(i+k)-1 \\ m\end{array}\right)\right]$

Therefore, equation (34) can be used to obtain some mathematical properties of the GT$\mathrm{Kw}$ order statistics. 


\section{Maximum Likelihood Estimator (MLE)}

Let $X_{1}, X_{2}, \ldots, X_{n}$ denote the random samples drawn from the GT-Kw distribution with observed values $x_{1}, x_{2}, \ldots ., x_{n}$. Let $\Theta=(a, b, c, d, \lambda)^{T}$ be the px1 parameter vector, to obtain the MLE of $\Theta$, the pdf of (7) can also be written as

$$
f(x)=c d x^{c-1} p^{d-1} y^{a-1} z
$$

where $p=\left(1-x^{c}\right), y=1-\left(1-x^{c}\right)^{d}, z=a(1+\lambda)-\lambda(a+b) y^{b}$. The likelihood function of (35) is given by

$$
\ell\left(x_{1}, x_{2}, \ldots, x_{n} / \Theta\right)=\prod_{i=1}^{n} f\left(x_{i}, \Theta\right)
$$

The log-likelihood of (36) is given by

$$
\ell=\ell(\Theta)=n \log c+n \log d+(c-1) \sum_{i=1}^{\infty} \log x_{i}+(d-1) \log \sum_{i=1}^{\infty} p_{i}+(a-1) \log \sum_{i=1}^{\infty} y_{i}+\log \sum_{i=1}^{\infty} z_{i}
$$

The sample statistic that maximizes the likelihood function $\ell(\Theta)$ is called the maximum

likelihood estimator of $\Theta$ and is denoted by $\Theta$. The ML Estimator of the GT-Kw model will be obtained after differentiating (37) with respect to each parameter a, b, $c, d, \lambda$ and setting the result to zero.

\section{Conclusion}

In this paper, we proposed and study a new model, called generalized transmuted Kumaraswamy model based on the GT - G family studied by Nofal (2017). We provided an explicit expression for the survival and hazard functions. The statistical properties including moments, moment generating function, incomplete moments, characteristics function and order statistics were derived. We discuss maximum likelihood estimation by using maximum method. We hope that the proposed model will attract wider application especially in hydrology to model hydrological data and related areas.

\section{Acknowledgement:}

The authors would like to thank the editor in chief and the reviewers for their helpful comments which improved upon the quality of the research.

\section{References}

1. Ahmad, A., Ahmad, S. P., Ahmed, A. (2015). Characterization and estimation of transmuted Kumaraswamy distribution, Mathematical Theory and Modeling, 5(9), 168-174.

2. Aryal, G.R., and Tsokos, C.P. (2011). Transmuted Weibull distribution: A generalization of Weibull probability distribution. European Journal of Pure and Applied Mathematics. 4, 89-102.

3. Cordeiro, G. M., Ortega, E. M. M., Nadarajah, S., (2010). The Kumaraswamy Weibull distribution with application to failure data. J Frankl Inst 347, 13991429.

4. Cordeiro, G. M., Nadarajah, S., Ortega, E. M. M., (2012). The Kumaraswamy Gumbel distribution, Stat Methods Appl 21: 139-168. 
5. Elbatal, I. (2013). Transmuted generalized Inverted Exponential Distribution. Eco. Qual. Control. 28(2): 125-133.

6. Fletcher, S. C. and Ponnambalam, K. (1996). Estimation of reservoir yield and storage distribution using moments analysis. Journal of Hydrology 182, 259-275.

7. Ganji, A., Ponnambalam, K. and Khalili, D. (2006). Grain yield reliability analysis with crop water demand uncertainty. Stochastic Environmental Research and Risk Assessment 20, 259-277. MR2297440.

8. Gupta, R.C., Gupta, P.I. and Gupta, R.D. (1998). Modeling failure time data by Lehmann alternatives. Communications in Statistics Theory and Methods, 16(27): 884-904.

9. Ishaq A. I., Usman A. and Usman A. U. (2018). On the generalized transmuted Rayleigh distribution. Benin Journal of Statistics, (1), 75-86.

10. Jones, M. C. (2009). Kumaraswamy's distribution: A beta-type distribution with some tractability advantages. Statistical Methodology 6, 70-81. MR2655540

11. Kumaraswamy, P. (1980). A Generalized probability density functions for double-random processes. Journal of hydrology, 46 (1-2): 79-88.

12. Nofal, Z. M., Afify, A. Z., Yousof, H. M. and Cordeiro, G. M. (2017). The generalized transmuted-G family of distributions. Communications in Statistics Theory and Methods. 46, 4119-4136.

13. Nofal, Z. M., Nofal, EL Gebaly Y. M. (2017). The Generalized Transmuted Weibull Distribution for Lifetime Data. Pak.j.stat.oper.res. Vol.XIII No.2 pp355378.

14. Ponnambalam, K., Seifi, A. and Vlach, J. (2001). Probabilistic design of systems with general distributions of parameters. International Journal of Circuit Theory and Applications 29, 527-536.

15. Seifi, A., Ponnambalam, K. and Vlach, J. (2000). Maximization of manufacturing yield of systems with arbitrary distributions of component values. Annals of Operations Research 99, 373-383. MR1837747

16. Shaw, W. T and Buckley, I. R. C. (2007). The alchemy of probability distributions: beyond Gram-Charlier expansionsand a skew-kurtotic-normal distribution from a rank transmutation map. arXiv preprint arXiv:0901.0434.

17. Sundar, V. and Subbiah, K. (1989). Application of double bounded probability density-function for analysis of ocean waves. Ocean Engineering 16, 193-200.

18. Usman A., Ishaq A. I., Umar K. A., Usman A. U. and Yakubu A. (2018). Generalized transmuted - generalized Rayleigh distribution: Its properties and Application. Journal of Experimental Research Statistics, 6(4), 77-84. 